\title{
The Clinical Profile and Ocular Manifestations of Herpes Zoster Ophthalmicus - A Hospital Based Study
}

\author{
Prathibha Shanthaveerappa, Remya Joseph Parappallil \\ Department of Ophthalmology, Rajarajeswari Medical College and Hospital, Rajiv Gandhi University of Health And Sciences, Bangalore, \\ India
}

\author{
Email address: \\ prathibhas@rediffmail.com (P. Shanthaveerappa), remyajosephparappallil@gmail.com (R. J. Parappallil)
}

\section{To cite this article:}

Prathibha Shanthaveerappa, Remya Joseph Parappallil. The Clinical Profile and Ocular Manifestations of Herpes Zoster Ophthalmicus - A Hospital Based Study. International Journal of Ophthalmology \& Visual Science. Vol. 4, No. 1, 2019, pp. 19-23.

doi: $10.11648 /$ j.ijovs.20190401.14

Received: January 28, 2019; Accepted: March 22, 2019; Published: April 18, 2019

\begin{abstract}
Background: Herpes Zoster Ophthalmicus (HZO) occurs due to reactivation of latent varicella zoster virus within the gasserian ganglion involving the ophthalmic division of the trigeminal nerve. HZO often has a chronic course with significant ocular morbidity as eye is considered potentially serious of all sites of herpes zoster owing to its delicate nature. Purpose:1. To study the mode of presentation, ocular manifestations and complications of herpes zoster ophthalmicus (HZO) 2. To analyse the predisposing factors for the development of HZO. Materials and Methods: A prospective clinical study was done in 20 patients who were clinically diagnosed with HZO in the outpatient department of ophthalmology over a period of one year. They were subjected to a detailed general and ocular examination and were treated medically with close follow up. Result: Advancing age was the most common risk factor. Acute neuralgia was the commonest presenting symptom (75\%). Ocular involvement was seen in 16 patients with no bilaterality. Conjunctiva $(60 \%)$ was the most common ocular structure involved followed by Cornea (45\%). Anterior uveitis $(20 \%)$ was complicated by haemorrhagic uveitis and orbital apex syndrome with total external ophthalmoplegia. Post herpetic neuralgia was the commonest complication seen. Conclusion: The potential manifestations of HZO are myriad. Development of serious inflammatory complications was associated with delay in therapy. Hence timely diagnosis and management are critical in limiting ocular morbidity
\end{abstract}

Keywords: Herpes Zoster Ophthalmicus (HZO), Acyclovir, Orbital Apex Syndrome, Post Herpetic Neuralgia

\section{Introduction}

Herpes Zoster (HZ) results from the reactivation of the varicella zoster virus which remains latent in the primary sensory ganglion like Gasserian ganglion. HZ involving the Ophthalmic division of the Trigeminal nerve is called Herpes Zoster Ophthalmicus (HZO), irrespective of the presence or absence of ocular involvement [1-3] . It usually manifests as a unilateral painful skin rash in a dermatomal distribution of the trigeminal nerve shared by the eye and ocular adnexa. This name is derived from the Latin word 'cingulum' which means girdle or belt because of its distribution along a single dermatome. HZO accounts for $10-25 \%$ of all herpes zoster cases $[4,5]$. Up to $20 \%$ of the population will have $\mathrm{HZ}$ at some time in life. While HZO does not necessarily affect the structures of the eye, many of the acute and long-term complications associated with the disease are the result of direct viral toxicity to the eye or the ensuing inflammatory response within the eye [6]. The frontal branch is most often involved in the ophthalmic division of the trigeminal. Approximately $50-72 \%$ of the patients with periocular zoster will have ocular involvement and sustain a moderate to severe degree of visual loss [7].

\section{Disease Entity}

\subsection{Risk Factors}

Risks for reactivation include any decline in the T-cell mediated immune response including that caused by normal aging, HIV/AIDS, and immunosuppressive medications [6]. Others include sex $(\mathrm{F}>\mathrm{M})$, white ethnicity, mechanical trauma, psychologic stress, organ transplant recipients and exposure to infected individuals [8]. The risk of herpes zoster 
is 15 times greater in men with HIV than in men without HIV [9].

\subsection{Course of the Disease}

\subsubsection{Pathophysiology}

HZO is caused by the human herpesvirus 3 , the same virus that causes varicella (chickenpox) belonging to the family Herpesviridae. Reactivation of the latent virus in neurosensory ganglia produces the characteristic manifestations of herpes zoster which replicates in the nerve cells, and sheds virions from the cells that are carried down the axons to the skin served by that ganglion [10].

\subsubsection{Clinical Features}

Classically, HZO begins with flu-like symptoms including fever, myalgia, and malaise for approximately one week. Typically, patients then develop a painful unilateral dermatomal rash in the distribution of one or more branches of trigeminal nerve: frontal, lacrimal and nasocilliary. The skin manifestations usually begin as an erythematous macular rash, progressing over several days into papules, vesicles, and then pustules which eventually ruptures and scabs over the course of two to three weeks [6].

Clinical manifestations (Table 1) of HZO can be caused by direct viral invasion, secondary inflammation and changes to the autoimmune mechanisms, and neurotrophic disorders [11]. Classically, involvement of the tip of the nose (Hutchinson's sign) has been thought to be a clinical predictor of ocular involvement. Although patients with a positive Hutchinson's sign have twice the incidence of ocular involvement, one third of patients without the sign develop ocular manifestations [12]. Reported complications of HZO include lid vesicles and scarring, several forms of conjunctivitis and keratitis, episcleritis, scleritis, uveitis, secondary glaucoma, papillary abnormalities, acute retinal necrosis, optic neuritis, central retinal artery occlusion, cranial nerve palsies ( $\mathrm{III}>\mathrm{VI}>\mathrm{IV}$ ), orbital apex syndrome, localized arteritis and post herpetic neuralgia [13].

Table 1. Clinical manifestations of HZO Mechanism 11.

\begin{tabular}{l|l}
\hline Direct viral invasion & Conjunctivitis, superficial keratitis (punctate keratitis, dendritic keratitis) \\
Secondary inflammation or alteration of autoimmune mechanism & Stromal keratitis, scleritis, episcleritis, uveitis \\
Neurotrophic changes & Neurotrophic keratitis
\end{tabular}

Neurotrophic changes $\quad$ Neurotrophic keratitis

With the above background, the present study was undertaken with the following objectives:

1) To study the mode of presentation, ocular manifestations and complications of HZO in a suburban population.

2) To analyse the predisposing factors for the development of $\mathrm{HZO}$

\section{Materials and Methods}

\subsection{Data Collection}

The study was a prospective hospital based study. All patients who attended the outpatient department of ophthalmology who were clinically diagnosed with HZO were studied over a period of 1 year.

\subsection{Methodology}

A standard clinical proforma, which included history, clinical findings and laboratory investigations, was used in all cases. Those patients in whom diagnosis of HZO was uncertain and healed cases of HZO were excluded from the study. A detailed ophthalmic examination including adnexal examination, ocular motility, Snellen best corrected visual acuity (BCVA), slit-lamp examination, corneal sensitivity and fluorescein testing. Grading of intraocular inflammation using standardization of uveitis nomenclature (SUN) working group criteria [14], non contact tonometry, and dilated fundus examination was also performed for all patients. Investigations like bloodsugar, HIV serology and complete hemogram were done in all patients. All patients were treated medically. The patients were treated with oral Acyclovir 800mg 5 times/day for 14 days and systemic non-steroidal anti-inflammatory drugs like Diclofenac or Ibuprofen. The skin lesions were treated with cool compresses and topical antibiotic ointment. Patients with only conjunctivitis were treated with topical antibiotic eye drops. Patients with epithelial keratitis were treated with topical Acyclovir 3\% eye ointment 5 times/day, prophylactic topical antibiotics and cycloplegics. Patients with uveitis received topical steroids and topical cycloplegics that were tapered according to the clinical response. In patients with raised IOP, Timolol $0.5 \%$ was added. Patients were followed up at regular intervals depending on the severity of involvement and the response to treatment was evaluated.

\subsection{Statistical Analysis}

Data entry and statistical analysis were performed using Windows-based SPSS statistical software. The data collected was analyzed statistically using descriptive statistics like frequency and percentage and was represented in tables.

\section{Results}

The clinical data of 20 patients presenting with features of HZO were evaluated.

In this study, it was found that the maximum incidence of HZO was in the age group of 51-60 years (35\%). The mean age was 49.5 with the youngest being $14 \mathrm{yrs}$ and oldest being $75 \mathrm{yrs}$. The age distribution is shown in table 2. Gender distribution 
Table 2. Age wise distribution of cases.

\begin{tabular}{ll}
\hline Age in years & No. of cases (\%) \\
\hline $11-20$ & $2(10 \%)$ \\
$21-30$ & $2(10 \%)$ \\
$31-40$ & $1(5 \%)$ \\
$41-50$ & $3(15 \%)$ \\
$51-60$ & $7(35 \%)$ \\
$61-70$ & $4(20 \%)$ \\
$71-80$ & $1(5 \%)$ \\
\hline
\end{tabular}

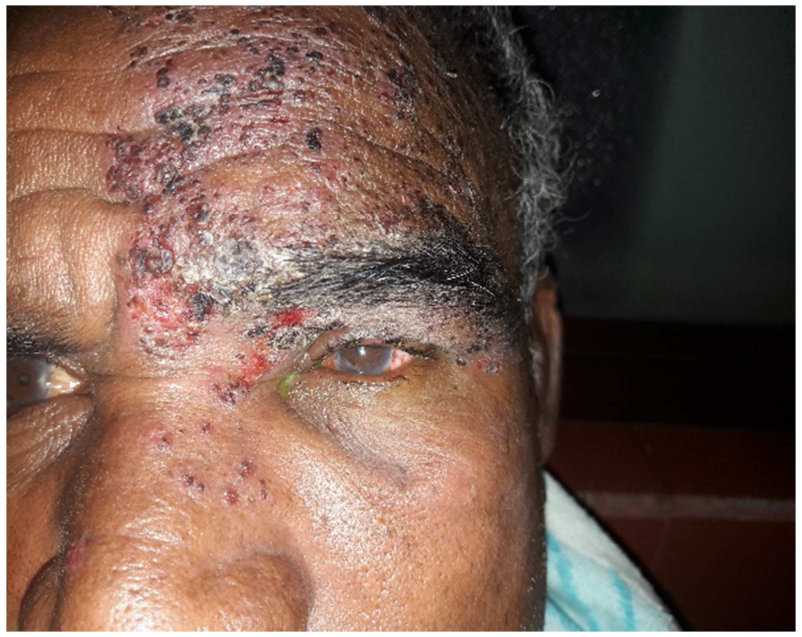

Figure 1. Eruptive skin lesions showing dermatomal distribution.

Males (65\%) dominated this study with M:F ratio of 1.8:1. The most common predisposing factor for the development of HZO was age more than 50 years $(60 \%)$. None of the patients were HIV seropositive. Skin lesions and acute neuralgia were the most common presenting symptoms which were present in all of the patients studied (100\%). Ocular involvement was seen in 16 patients $(80 \%)$ while 4 had only skin lesions. The clinical profile is summarized in table 3 .

Table 3. Sex distribution, Predisposing factors, Ocular involvement and presenting features.

\begin{tabular}{llll}
\hline & & No of cases & percentage \\
\hline Gender distribution & Male & 13 & $65 \%$ \\
& Female & 7 & $35 \%$ \\
Predisposing & Age $>$ 50yrs & 12 & $60 \%$ \\
factors & diabetes & 9 & $45 \%$ \\
& No predisposing & 6 & $30 \%$ \\
& factors & & \\
Ocular & HIV & 0 & \\
involvement & Present & 16 & $80 \%$ \\
& Absent & 4 & $20 \%$ \\
Presenting & Acute neuralgia & 20 & $100 \%$ \\
symptoms & Skin rashes & 20 & $100 \%$ \\
& Watering & 10 & $50 \%$ \\
& Lid swelling & 8 & $40 \%$ \\
& Diminution of & 5 & $25 \%$ \\
\hline
\end{tabular}

The conjunctiva stood out as the most common ocular structure involved ( $75 \%$ of cases) followed by the cornea (in $56 \%$ cases). Anterior uveitis was seen in 4 patients of which 1 presented with hemorrhagic uveitis with hypopyon. Orbital apex syndrome with complete external ophthalmoplegia was seen in 1 patient. The ocular structures involved are summarized in table 4 .

Table 4. Ocular structures involved in HZO.

\begin{tabular}{llll}
\hline Serial no & Ocular structures involved & No of patients & percentage \\
\hline 1 & Lids & 8 & $50 \%$ \\
2 & Conjunctiva & 12 & $75 \%$ \\
3 & Cornea & 9 & $56 \%$ \\
4 & Episclera and sclera & 0 & 0 \\
5 & Uveal tract & 4 & $25 \%$ \\
6 & Secondary glaucoma & 5 & $32 \%$ \\
7 & Lens & 2 & $12 \%$ \\
8 & Extraocular muscles & 1 & $6 \%$ \\
\hline
\end{tabular}

Post herpetic neuralgia (40\%) was the most common complication noted at 1 month follow up while 7 patients (35\%) recovered without any sequelae. The ocular complications are given in table 5.

Table 5. Ocular complications seen among HZO patients in our study

\begin{tabular}{llll}
\hline Serial no & Complications & No. of patients & Percentage \\
\hline 1 & Post herpetic neuralgia & 8 & $40 \%$ \\
2 & Lid scarring & 6 & $30 \%$ \\
3 & Follicular conjunctivitis & 3 & $15 \%$ \\
4 & Punctate epithelial keratitis & 3 & $15 \%$ \\
5 & Disciform keratitis & 2 & $10 \%$ \\
6 & Dendritic ulcer & 1 & $5 \%$ \\
7 & Secondary glaucoma & 3 & $15 \%$ \\
8 & Persistent synechiae with corneal vascularisation & 1 & $5 \%$ \\
9 & Ptosis & 1 & $5 \%$ \\
\hline
\end{tabular}




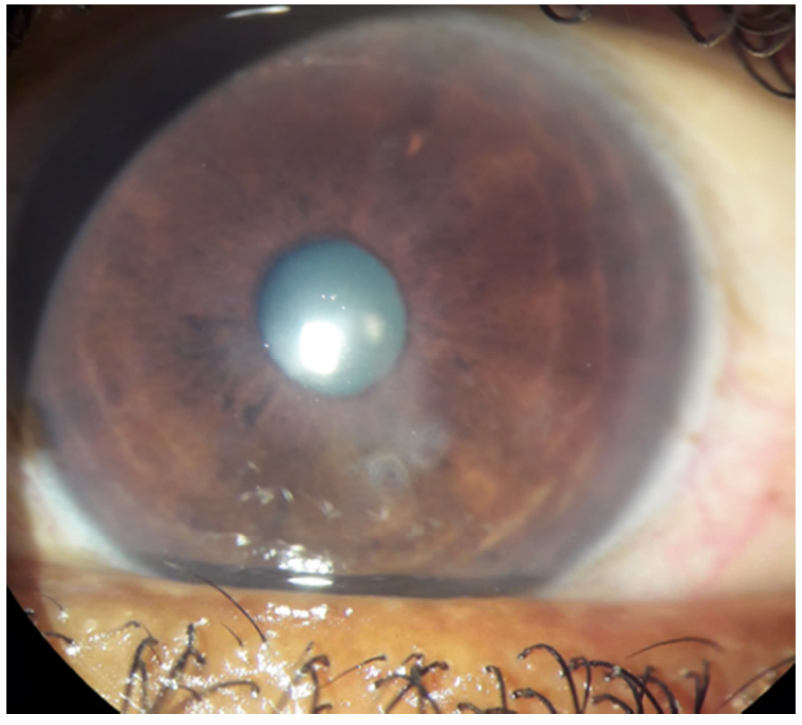

Figure 2. Stromal keratitis.

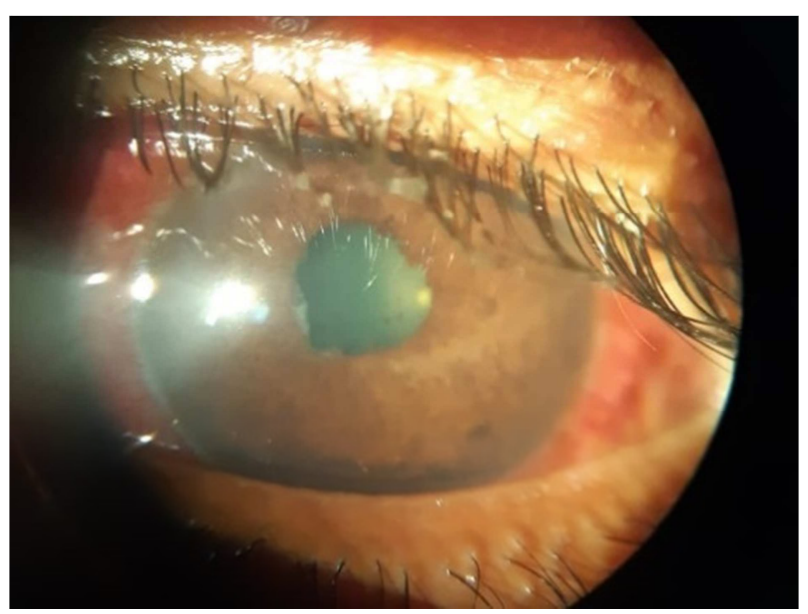

Figure 3. Posterior synechiae with iris atrophy.

\section{Discussion}

HZO is a very painful and debilitating ocular disease causing substantial visual loss and socioeconomic disability . It was observed in this study that HZO occurred maximally in the fifth to sixth decade of life (55\%) suggesting that advancing age is the most important predisposing factor for development of HZO.

A recent case series reports that HZO affects similarly individuals aged younger than or older than 60 years, with the most common decade of onset between age 50 and 59 years [15]. In a recent study, the incidence rate for the subgroup of the population older than 65 years was approximately five times that of the rest of the population [5, 16]. Increased age has been associated with a decrease in cell-mediated immunity, which is a crucial factor to avoid reactivation of the latent varicella zoster virus.

Male dominated this study (M:F- 1.8:1)which was similar to Malik et al. [17] study and study from Ethiopia 19] in contrast to Prabhu et al. [18]study which showed female preponderance. The higher rate of $\mathrm{HZO}$ among male patients perhaps reflects the fact that males have better access to health care and report early.

HZO was found to be an early clinical marker of HIV infection especially in patients aged $<45$ years. The study in Ethiopia [19] supported this finding which showed $95.3 \%$ of total population and $100 \%$ of patients aged $<45$ years were HIV seropositive. But in the present study, none of the patients were tested positive for HIV infection. In the present study, ocular involvement was seen in 16 patients $(80 \%)$ which was similar to Liesengang et al [5] study. Bilateral presentation, a feature of disseminated zoster was not seen in this study.

Among the various ocular structures involved, conjunctival involvement $(60 \%)$ was the most common in the form of conjunctivitis followed by cornea (45\%).

Corneal involvement in the form of absent or reduced sensation was noted in 6 patients $(37 \%), 5$ patients had punctate epithelial keratitis (31\%), 2 had stromal keratitis $(12.5 \%)$ and 1 had pseudodentrite formation (6\%). This was lesser than that observed in studies from the Ethiopia and Liesegang et al which were both $65 \%$ but was close to study from the United Kingdom (49\%) [20]. The prevalence of ophthalmoplegia was reported as $3.5-10.1 \%$ in the two large HZO case series in the literature [20,21].

Post herpetic neuralgia (30\%) was the commonest complication noted at 1 month follow up followed by lid scarring $(25 \%)$ which was comparable to study from Ethiopia [19]. It has been also shown that patients with keratitis, conjunctivitis, or uveitis had a higher risk of developing PHN compared with patients who did not have these ocular features [16].

It was observed that 9 patients with HZO (45\%) in whom oral Acyclovir was started within 72 hours of onset of skin rash recovered completely. This correlates with prospective controlled clinical trials which have reported a beneficial effect of Acyclovir on ocular complications of HZO [22].

\section{Conclusion}

The present study outlines the clinical profile of HZO that can virtually affect any ocular structures. The management of HZO usually involves a multidisciplinary approach aiming to reduce both complications and ocular morbidities. Antiviral medications like Acyclovir, Valacyclovir and Famciclovir remain the mainstay of therapy and are effective in preventing serious ocular complications of $\mathrm{HZO}$ when begun within 72 hours of onset of skin rash. However further studies are required to guide therapeutic approaches to the chronicity and recurrence of HZO.

In the future, a reduction in the incidence and severity of HZO may result from a more widespread use of Varicella vaccine (zostavax vaccine) in an effort to obtain herd immunity. Eye drops containing tetra peptides derived from substance $P$ and insulin-like growth factor-1 have demonstrated rapid epithelial healing of corneal defects and regeneration of corneal nerve fibers, renewing corneal 
sensitivity and reducing incidence of corneal hypoesthesia. In addition, sterile eye drops containing thymosin b4 have been reported to reduce geographic defects and reduce ocular irritation in patients affected by herpes zoster ophthalmicus.

\section{References}

[1] Kanski JJ., Cornea, Chapter 5. In: Clinical Ophthalmology. 5th edition., (Edinburgh: Butterworth Heinemann; 2003). p111-114.

[2] Wilson FI. Varicella and Herpes Zoster ophthalmicus. Chap. 25 In : Tabbara K, Hyndiuk R eds. Infections of the eye 2nd edition. ( Bosten: Little, Brown, 1996):387-400.

[3] Deborah Pavan-Langston. Herpes Zoster Ophthalmicus. Neurology 1995; 45(suppl 8): S50-S51.

[4] Ragozzino MW, Melton LJ 3d, Kurland LT, Chu CP, Perry HO. Population-based study of herpes zoster and its sequelae. Medicine. 1982; 61:310-6.

[5] Liesegang TJ. Herpes Zoster Ophthalmicus. Ophthalmology 2008; 115: S3-S12.

[6] Thomas Catron, MD and H. Gene Hern, MD, West J Emerg Med. 2008 Aug; 9(3): 174-176.

[7] Deborah Pavan-Langston. Viral diseases of the ocular anterior segment. Chap 14. In: Foster CS., Azar DT., Dohlman CH.eds. Smolin and Thoft's. The cornea. Scientific foundations and clinical practice. 4th edn. (Philadelphia: Lippincott Williams and Wilkins 2005); p297-397.

[8] Thomas SL, Hall AJ. What does epidemiology tell us about risk factors for herpes zoster? Lancet Infect Dis 2004; 4(1):2633.

[9] Buchbinder SP, Katz MH, Hessol NA, et al. Herpes zoster and human immunodefciency virus infection. J Infect Dis 1992; 166:1153-1156.

[10] Evaluation and Management of Herpes Zoster Ophthalmicus SAAD SHAIKH, M. D., and CHRISTOPHER N. TA, M. D., Stanford University Medical Center, Stanford, California Am Fam Physician. 2002 Nov 1; 66(9):1723-1730.

[11] Colin J, Prisant O, Cochener B, et al. Comparison of the efficacy and safety of valacyclovir and acyclovir for the treatment of herpes zoster ophthalmicus. Ophthalmology 2000; 107: 1507-1511.

[12] Harding SP, Lipton JR, Wells JC. Natural history of herpes zoster ophthalmicus: predictors of postherpetic neuralgia and ocular involvement. Br J Ophthalmol. 1987; 71:353-8.

[13] Christopher E. Starr., Deborah Pavan-Langston. Varicella Zoster virus: Mechanisms of pathogenicity and corneal disease. Ophthalmol Clin N Am. 2002; 15:7-15.

[14] Jabs DA, Nussenblatt RB, Rosenbaum JT; Standardization of Uveitis Nomenclature (SUN) Working Group (2005) Standardization of uveitis nomenclature for reporting clinical data. Results of the first international workshop. Am J Ophthalmol 140:509-516.

[15] Ghaznawi N, Virdi A, Dayan A, Hammersmith KM, Rapuano CJ, Laibson PR, Cohen EJ (2011) Herpes zoster ophthalmicus: comparison of disease in patients 60 years and older versus younger than 60 years. Ophthalmology 118:2242-2250.

[16] Borkar DS, Tham VM, Esterberg E, Ray KJ, Vinoya AC, Parker JV, Uchida A, Acharya NR (2013) Incidence of herpes zoster ophthalmicus: results from the Pacific ocular inflammation study. Ophthalmology 120:451-456.

[17] Malik LM, Azfar NA, Khan AR, et al. Herpes zoster in children. J Pak Assoc Dermatologists 2013; 23(3):267-271.

[18] Prabhu S, Sripathi H, Gupta S, et al. Childhood herpes zoster: a clustering of ten cases. Indian J Dematol 2009; 54(1):62-64

[19] Bayu S, Alemayehu W. Clinical Profile of Herpes zoster ophthalmicus in Ethiopians. Clin Infect Dis. 1997; 24:125660 .

[20] Marsh RJ, Cooper M. Acyclovir and steroids in herpes zoster Keratouveitis. Br J Ophthal 1984; 68(12):904-905.

[21] Womack LW, Liesegang TJ. Complications of herpes zoster ophthalmicus. Arch Ophthalmol. 1983; 101:42-5.

[22] Wood M J., Shukla S., Fiddian AP., Crooks RJ. Treatment of acute herpes zoster : Effect of early versus late therapy with Acyclovir and Valaciclovir on prolonged pain. J Infect Dis 1998; 178(Suppl 1): s81-s84. 